\title{
Nutrição para os praticantes de exercício em grandes altitudes
}

\author{
Nutritional strategy for exercising in high altitudes
}

\section{R E S U M O}

Caroline BUSS

Álvaro Reischak de OLIVEIRA²

Quando o atleta ascende a uma grande altitude, ele é exposto a uma pressão barométrica reduzida, e os efeitos fisiológicos que acompanham estas mudanças da pressão atmosférica podem ter grande influência sobre o seu organismo e seu desempenho físico. Acredita-se que a hipóxia seja responsável pelo início de uma cascata de eventos sinalizadores que, ao final, levam à adaptação à altitude. A exposição aguda à hipóxia provoca sonolência, fadiga mental e muscular e prostração. Cefaléia, náusea e anorexia são sintomas provocados pela Doença Aguda das Montanhas, que pode ocorrer nos primeiros dias de permanência na altitude. Uma estratégia nutricional adequada é fundamental para que o organismo não sofra nenhum estresse adicional. O objetivo deste trabalho foi apresentar os principais efeitos da altitude sobre o organismo e sobre o desempenho físico, discutir e/ou sugerir recomendações nutricionais para esta situação e, se possível, apresentar uma orientação nutricional prática para o atleta na altitude. Algumas das principais conclusões encontradas foram: o consumo energético deve ser aumentado; é fundamental monitorar a quantidade de líquidos ingeridos e escolher alimentos agradáveis ao paladar, ricos em energia e nutrientes. Recomenda-se trabalhar com um nutricionista do esporte com antecedência, para que um plano alimentar individual seja elaborado e colocado em prática antes mesmo da viagem à altitude.

Termos de indexação: altitude; exercício; nutrição esportiva; planejamento alimentar.

\section{A B S T R A C T}

When athletes are subject to high altitudes, they are exposed to a lower barometric pressure and the physiological effects that accompany these atmospheric pressure changes can have a strong influence on their bodies and performance. Hypoxia is thought to be responsible for triggering a cascade of signaling events that eventually leads to altitude acclimatization. Acute exposure to hypoxia causes sleepiness, mental and muscle fatigue and prostration. Headache, nausea and anorexia are some of the symptoms of Acute Mountain Sickness which may occur in the first days of high altitude sojourn. An adequate nutritional strategy is essential to protect the body from any additional stress. The aim of this paper was to present the main effects of altitude on the

\footnotetext{
1 Curso de Nutrição, Faculdade de Medicina, Universidade Federal do Rio Grande do Sul. Rua Ramiro Barcelos, 2400, $4^{\mathrm{a}}$ andar, Rio Branco, 90035-003, Porto Alegre, RS, Brasil. Correspondência para/Correspondence to: C. BUSS.

2 Departamento de Desportes, Escola Superior de Educação Física, Universidade Federal do Rio Grande do Sul. Porto Alegre, RS, Brasil.
} 
human body and physical performance; to discuss and/or suggest nutritional recommendations for this situation; and, if possible, to present practical nutritional guidelines for athletes in high altitudes. Some of the main conclusions found were: energy intake must be increased; it is essential to monitor fluid intake and to choose palatable energy and nutrient-dense foods. It is recommended to work with a sports dietitian in advance, so that an individual nutrition plan can be made and put into practice even before being exposed to high altitudes.

Indexing terms: altitude; exercise; sports nutrition; food planning.

\section{N T R O D U Ç Ã O}

Parte do relevo do planeta está acima de uma altura de três mil metros, uma designação arbitrária que separa as altitudes moderada e alta'. Embora o número de pessoas que realmente vivam nessa elevação seja pequeno, milhares de pessoas viajam para localidades em grandes altitudes, seja para trabalho ou lazer, expondo-se aos efeitos da pressão barométrica reduzida.

As estatísticas do Parque Aconcagua, localizado na Argentina, demonstram o aumento do número de visitantes ano a ano, chegando a um total de 6994 visitantes durante a temporada 2003 e 2004, sendo que, aproximadamente, $60 \%$ destes envolveram-se em atividades de escalada².

No caso do atleta, a finalidade do treinamento na altitude é de preparação específica para uma competição, ou para submeter o organismo a um estresse fisiológico adicional em um determinado ponto do macrociclo de treinamento ${ }^{3}$.

A pressão barométrica se modifica em função da altitude, e as características físicas e efeitos fisiológicos que acompanham as mudanças da pressão podem ter grande influência sobre o desempenho físico ${ }^{4}$. Apesar da pressão diminuir com o aumento da altitude, as porcentagens dos gases que compõem o ar permanecem as mesmas. Assim, com uma diminuição da pressão barométrica, a pressão parcial de oxigênio inspirada $\left(\mathrm{PIO}_{2}\right)$ irá diminuir proporcionalmente.

A exposição à hipóxia traz riscos associados ao organismo ${ }^{5}$. Sonolência, fadiga mental e muscular, prostração, cefaléia e, ocasionalmente, náusea são alguns dos efeitos agudos importantes da hipóxia. Há, também, o risco associado de Doença Aguda das Montanhas (DAM) e, com menor freqüência, de edemas pulmonar e cerebral. Os sintomas da DAM incluem dor de cabeça, náusea, anorexia e fadiga, ocorrendo principalmente em pessoas que ascendem rapidamente a grandes altitudes ${ }^{1,6}$.

Acredita-se que a hipóxia seja responsável pelo início de uma cascata de eventos sinalizadores que, ao final, levam à adaptação à altitude$^{1}$. A aclimatação ocorre pelos seguintes meios: (I) grande aumento da ventilação pulmonar - $65 \%$ acima do normal - pela estimulação hipóxica dos quimioreceptores, (II) aumento do hematócrito, de valores normais de 40 a $45 \mathrm{~g} / \mathrm{dl}$ até $60 \mathrm{~g} / \mathrm{dl}$ e da concentração de hemoglobina, de valores normais de $15 \mathrm{~g} / \mathrm{dl}$ até $20 \mathrm{~g} / \mathrm{dl}$, (III) vascularização aumentada dos tecidos e (IV) capacidade aumentada das células de utilização de oxigênio ${ }^{3,4}$.

Estudos mostram que os efeitos da altitude e as adaptações fisiológicas que induzem à aclimatação provocam modificações metabólicas - como a alteração da utilização de substrato, da capacidade de processamento de nutrientes - e físicas - como a perda de massa corporal ${ }^{7-14}$. A alimentação torna-se, então, fundamental na tentativa de prevenir ou minimizar as conseqüências adversas da altitude sobre o indivíduo.

O objetivo deste trabalho foi trazer uma visão geral dos principais efeitos da altitude sobre o organismo e sobre o desempenho físico, discutir e/ou sugerir recomendações nutricionais para esta situação e, se possível, apresentar uma orientação nutricional prática para o atleta na altitude. 


\section{Efeitos da altitude sobre o organismo e o desempenho físico}

O desempenho no exercício, em condições de pressão atmosférica reduzida, é afetado pela influência de três fatores: a resistência do ar, a pressão parcial de oxigênio, e o processo de aclimatação, que afeta o transporte de oxigênio, o metabolismo e o balanço ácido-básico. O impacto sobre o desempenho varia conforme a modalidade do exercício e a distância e duração da prova. Há também uma grande variação individual entre atletas no que diz respeito à forma como são afetados por uma mudança aguda na $\mathrm{PIO}_{2}$ e como se adaptam a uma nova pressão atmosférica com a exposição crônica ${ }^{15-17}$.

O processo de aclimatação na altitude leva de duas a três semanas, resultando em adaptações sistêmicas que podem ser medidas como respostas fisiológicas ${ }^{18}$. Estas adaptações - como o aumento na ventilação, na concentração de hemoglobina, na densidade capilar, no número de mitocôndrias e na concentração de mioglobina tecidual - melhoram o transporte de oxigênio. Ao final do período de aclimatação, essas adaptações fisiológicas ocorridas estão relacionadas com a melhora do desempenho de resistência aeróbica na altitude ${ }^{19}$.

Atletas e técnicos devem levar em consideração que a intensidade de exercício que pode ser mantida pelo organismo diminui na altitude, o que traz implicações para competições e treinos $^{20}$. O resultado do treinamento na altitude depende do balanço entre a extensão da aclimatação do organismo e a habilidade do atleta em treinar numa intensidade suficientemente alta. Na altitude há um aumento na geração de lactato, uma queda do $\mathrm{pH}$ e uma taxa de fadiga aumen$\operatorname{tada}^{21}$ e, como conseqüência, a qualidade do estímulo do treinamento é reduzida, e muitos atletas podem até obter uma regressão do desempenho esportivo com o exercício em condições crônicas de hipóxia²2

As evidências sobre a questão altitude e desempenho são controversas. Estudos controlados com permanência na altitude ${ }^{23}$, ou simulação de altitude em câmara hipobárica ${ }^{24}$, demonstram melhora no desempenho, enquanto outros, com semelhante metodologia25,26, concluem que o treinamento em condições de hipóxia não provoca nenhum efeito aditivo no desempenho.

É necessário, assim, um maior embasamento científico, para que se comprove que a aclimatação à altitude provoca, de fato, mudanças no desempenho físico ao retornar para o nível do $\operatorname{mar}^{4}$. A investigação na área de nutrição, desempenho e altitude possui entraves, como a grande variabilidade da resposta individual ao treinamento na altitude e à exposição à hipóxia15-17,27, a dificuldade de formação de um grupo controle dos atletas estudados ${ }^{28}$, para assegurar que possíveis mudanças no desempenho tenham ocorrido pela presença da altitude e não pelo treinamento per se; e a própria permanência na altitude, que dificulta o estudo de grandes amostras.

Para que se possa demonstrar a manutenção do desempenho e a duração destes resultados após permanência em altitude, pesquisas futuras deveriam continuar investigando os efeitos deste tipo de treinamento após o retorno dos indivíduos ao nível do mar. Isso poderia permitir o estabelecimento de uma estratégia com a inclusão de um microciclo na altitude. Estudos com grupos cuidadosamente controlados, pareados, com intervenção randomizada, poderão superar grande parte das limitações acima citadas, elucidando, de forma mais consistente, a questão treinamento na altitude e desempenho.

\section{Alterações na utilização de substrato e na composição corporal}

Quando os atletas ascendem a grandes altitudes, pode ocorrer uma perda de peso corporal de até 3\% em 8 dias - em uma elevação de 4300 metros ou de até $15 \%$ após um período de 3 meses em uma altitude de 5300 a 8 mil metros ${ }^{20}$. Uma das causas deste fenômeno é a redução do apetite e consumo alimentar, decorrente dos efeitos da altitude sobre o organismo. Esta combinação pode exercer um efeito negativo sobre o desempenho, mesmo em altitudes moderadas, e 
trazer conseqüências secundárias, como consumo insuficiente de energia, depleção das reservas de glicogênio muscular, balanço de nitrogênio negativo e perda de massa corporal7-14.

Estudos demonstram o aumento da taxa metabólica basal na altitude ${ }^{14,29}$ e o consumo energético geralmente inferior, não atingindo a necessidade energética do indivíduo, que pode aumentar de 400 a $600 \mathrm{kcal} / \mathrm{dia}^{14,30}$. Uma exposição aguda a uma altitude de 4300 metros, por exemplo, eleva a taxa metabólica basal em torno de $30 \%$ e, mesmo após uma aclimatação de três semanas, ela permanece $17 \%$ mais alta que a taxa metabólica basal ao nível do mar $^{31}$.

As grandes altitudes resultam em adaptações fisiológicas a curto e longo prazo que afetam a necessidade e utilização de alguns nutrientes ${ }^{32}$. Em condições de equilíbrio do balanço energético e de nitrogênio, a aclimatação resulta em uma menor dependência de lipídios como substrato energético, tanto no repouso como em exercícios prolongados, e em uma dependência aumentada do metabolismo de glicose $\mathrm{e}^{7,9,33,34}$.

Um aumento do estresse oxidativo também é observado durante o exercício na altitude, mesmo sem um esforço físico máximo' ${ }^{1}$. São vários os fatores ambientais, além da hipóxia, que levam a tal condição, como variações da temperatura, intensidade aumentada da radiação ultravioleta e taxa metabólica aumentada ${ }^{35}$. Acredita-se que as espécies reativas de oxigênio, geradas no processo oxidativo - como os radicais superóxido $\left(\mathrm{O}_{2}^{-}\right)$e hidroxila $\left(\mathrm{OH}^{-}\right)$e o peróxido de hidrogênio $\left(\mathrm{H}_{2} \mathrm{O}_{2}\right)$ - iniciem importantes respostas de adaptação à altitude, entretanto, se produzidas em excesso, podem reduzir a perfusão capilar e prejudicar a função muscular na altitude ${ }^{1}$.

\section{Energia e macronutrientes}

Com a redução do apetite e consumo alimentar, juntamente com o aumento da taxa metabólica basal, torna-se mais difícil atingir a necessidade energética na altitude, que pode variar entre 3800 e $6 \mathrm{mil} \mathrm{kcal} / \mathrm{dia}$, dependendo do tipo de trabalho físico, sexo e idade do atleta ${ }^{14}$. A distribuição ideal do consumo energético entre os macronutrientes é controversa. A preferência por carboidratos foi mostrada em indivíduos que receberam uma variedade de alimentos ad libitum em altas altitudes e após atividade física aumentada ${ }^{8}$.

Uma dieta rica em carboidratos pode ser vantajosa ao atleta, pois o carboidrato é uma fonte de energia mais eficiente (maior produção de energia por litro de captação de oxigênio comparado à gordura: $5,05 \mathrm{kcal} / \mathrm{l} \mathrm{O}_{2}$ contra $4,69 \mathrm{kcal} / / \mathrm{O}_{2}$ ), independentemente da tensão de oxigênio no ar inspirado. Foi demonstrado, também, que o consumo de carboidratos melhora a oxigenação sangüínea na altitude, através do aumento da tensão de oxigênio e da saturação de oxihemoglobina no sangue arterial ${ }^{36}$.

Um estudo que avaliou o consumo dos diferentes macronutrientes em altitudes crescentes, entretanto, demonstrou uma tendência de aumento no consumo tanto de alimentos ricos em carboidrato como de alimentos ricos em gordura ${ }^{37}$. O mais recomendável, então, seria não excluir alimentos saborosos ricos em gordura, já que são fontes ricas em energia, que podem ajudar no fornecimento da necessidade energética aumentada na altitude. Queijo, peixes enlatados em óleo, chocolate, entre outros, preenchem os critérios de alimentos ricos em gordura, que são facilmente preparados para o consumo ${ }^{37}$.

Outro recurso para atingir a necessidade energética é com a suplementação de carboidratos através dos líquidos da dieta ${ }^{38}$. A partir da chegada na altitude, é recomendado consumir, no mínimo, de 3 a 5 litros de líquidos por dia, contendo de 200 a 300 gramas de carboidrato adicionais ${ }^{39}$. É fundamental manter o consumo de carboidratos durante o exercício, o que pode ser feito ingerindo uma bebida com $6 \%$ a $8 \%$ de carboidrato/ml e, na fase de recuperação pós-exercício, por meio do consumo de suplementos como bebidas energéticas ( $20 \%$ de carboidrato/ml), géis de carboidrato e barras energéticas.

Nenhum estudo demonstra que a recomendação de proteína no exercício $(1,2$ a 1,8g/kg de peso $)^{40}$ se altere na altitude. O balanço de 
nitrogênio negativo na fase aguda de exposição à altitude ocorre se houver um balanço energético negativo, e não devido a algum efeito da hipóxia sobre a digestibilidade e absorção da proteína ${ }^{31}$.

O padrão de alimentação na altitude também é alterado, devido à diminuição do apetite. Em um estudo de Westerterp-Platenga et al. ${ }^{8}$ foi demonstrado que o tamanho das refeições é reduzido, devido a um maior aumento na saciedade e diminuição da fome. Há, conseqüentemente, um aumento na freqüência de refeições de $4 \pm 1$ para $7 \pm 1$ vezes ao dia. Torna-se importante, então, a disponibilidade de alimentos fáceis de serem consumidos, ricos em energia e nutrientes.

\section{Hidratação}

O risco de desidratação pode, teoricamente, ser maior na altitude, devido à baixa umidade do ar, à diurese aumentada nas primeiras horas de exposição ${ }^{41}$ e ao aumento da ventilação pulmonar ${ }^{3}$. Na prática, no entanto, a perda total de água na altitude não é maior que ao nível do mar ${ }^{42,43}$. Um estudo mostra, ainda, que a perda total de água em relação ao nível do mar pode até diminuir em uma altitude de $4350 \mathrm{~m}$, de 4,5 para 3,5 litros/dia, respectivamente, devido à diminuição na temperatura ambiente e no consumo voluntário de líquidos ${ }^{44}$.

Recomenda-se o consumo em torno de 3 a 5 litros por dia ${ }^{32}$, já que a diurese é regulada em função da ingestão hídrica e a retenção de fluidos na altitude é uma das causas da DAM ${ }^{44}$. Uma forma prática de observar a hidratação é monitorar a urina. O organismo deve produzir urina de cor amarelo-pálida; se a urina apresentar-se concentrada e escura, é um indicativo de desidratação.

A adição de carboidratos nos líquidos promove a ingestão, pois aumenta a palatabilidade. Este é um aspecto importante já que a diminuição do apetite também vem acompanhada da diminuição da sensação de sede, e a hipóxia provoca mudanças nas respostas hedônicas, particularmente, um aumento da palatabilidade pelo gosto doce ${ }^{45}$.

\section{Micronutrientes}

A suplementação de vitaminas com função antioxidante poderia ser desejável em grandes altitudes devido ao estresse oxidativo aumentado ${ }^{35,46}$. Durante uma permanência prolongada na altitude, a suplementação de tais vitaminas poderia prevenir uma diminuição do desempenho físico, associada com o dano provocado pelos radicais livres aos sistemas de defesa celular ${ }^{39}$. Apenas um estudo, porém, sugere uma recomendação de suplementação ${ }^{47}$, sendo mais prudente aguardar que outros trabalhos sejam realizados a fim de melhor fundamentar a suplementação destes nutrientes.

Devido ao aumento da resposta eritropoiética na altitude, acredita-se que a suplementação de ferro pode ser benéfica para atletas com deficiência do mesmo, já que estes não demonstram uma resposta eritropoiética normal quando expostos à altitude ${ }^{48}$. É importante salientar, no entanto, que a produção simultânea de radicais livres pode ser aumentada por ferro livre em excesso ${ }^{49}$. Assim, mais evidências são necessárias para definir a necessidade e/ou quantidade da suplementação de ferro.

Tendo em vista o crescente número de pessoas que ascendem a grandes altitudes, seja a trabalho, no caso atleta, ou a lazer, é fundamental a continuidade das pesquisas na área, para que questões como a proporção ideal de macronutrientes, o risco de desidratação, e a necessidade de suplementação sejam elucidadas.

\section{O N C L US Ã O}

As grandes altitudes podem prejudicar o atleta pela combinação de vários efeitos, como a diminuição do apetite, mal-estar e náusea, que acabam por levar a uma perda de massa corporal. Assim, o consumo energético deve ser aumentado em aproximadamente 400 a $600 \mathrm{kcal} / \mathrm{dia}$; é fundamental monitorar a quantidade de líquidos ingeridos e incluir, no plano alimentar, itens de fácil preparação, agradáveis ao paladar e ricos em energia e nutrientes. 


\section{REFERÊ NCIAS}

1. Askew EW. Work at high altitude and oxidative stress: antioxidant nutrients. Toxicology. 2002; 180(2):107-19.

2. AconcaguaExpeditions.com [Internet]. Mount Aconcagua information, services and climbing and trekking expeditions - Mendoza - Argentina; c2004. Estatísticas. Disponível em: http:// www.aconcaguaexpeditions.com/ingles/2/ estadisticas.htm\#a

3. Townsend NE, Gore CJ, Hahn AG, McKenna MJ, Aughey RJ, Clark SA, et al. Living high-training low increases hypoxic ventilatory response of well trained endurance athletes. J Appl Physiol. 2002; 93(4):1498-505.

4. Bailey DM, Davies B. Physiological implications of altitude training for endurance performance at sea level: a review. Br J Sports Med. 1997; 31(3):183-90

5. Bailey DM, Castell LM, Newsholme EA, Davies B. Continuous and intermittent exposure to the hypoxia of altitude: implications for glutamine metabolism and exercise performance. Br J Sports Med. 2000; 34(3):210-2.

6. Hackett PH, Roach RC. High-altitude illness. N Eng J Med. 2001; 345(2):107-14.

7. Westerterp KR. Limits to sustainable human metabolic rate. J Exp Biol. 2001; 204(Pt18): 3183-7.

8. Westerterp-Plantenga MS, Westerterp KR, Rubbens M, Verwegen CRT, Richalet J-P, Gardette B. Appetite at 'high altitude' [Operation Everest III (Comex'97)]: a simulated ascent of Mount Everest. J Appl Physiol. 1999; 87(1):391-9.

9. Roberts AC, Butterfield GE, Cymerman A, Reeves JT, Wolfel EE, Brooks GA. Acclimatization to 4,300-m altitude decreases reliance on fat as a substrate. J Appl Physiol. 1996; 81(4):1762-71.

10. Vasankari TJ, Kujala UM, Rusko H, Sarna S, Athoupa $M$. The effect of endurance exercise at moderate altitude on serum lipid peroxidation and antioxidative functions in humans. Eur J Appl Physiol. 1997; 75(5):396-9.

11. Rawal SB, Singh MV, Tyagi AK, Roy J, Dimri GP, Selvamurthy W. Effect of time exposure to high altitude on zinc and copper concentrations in human plasma. Aviat Space Environ Med. 1999; 70(12):1161-5.

12. Fulco CS, Friedlander AL, Muza SR, Rock PB, Robinson S, Lammi E, et al. Energy intake deficit and physical performance at altitude. Aviat Space Environ Med. 2002; 73(8):758-65.
13. Bailey DM, Davies B, Milledge JS, Richards M, Williams SR, Jordinson $\mathrm{M}$, et al. Elevated plasma cholecystokinin at high altitude: metabolic implications for the anorexia of acute mountain sickness. High Alt Med Biol. 2000; 1(1):9-23.

14. Reynolds RD, Lickteig AJ, Deuster PA, Howard MP, Conway JM, Pietersma A, et al. Energy metabolism increases and regional body fat decreases while regional muscle mass is spared in humans climbing Mt. Everest. J Nutr. 1999; 129(7):1307-14.

15. Gore CJ, Hahn AG, Scroop GC, Watson DB, Norton $\mathrm{KI}$, Wood RJ, et al. Increased arterial desaturation in trained cyclists during maximal exercise at 580 m altitude. J Appl Physiol. 1996; 80(6):2204-10.

16. Stray-Gundersen J, Chapman RF, Levine BD. HiLo training improve performance in elite runners. Med Sci Sports Exerc. 1998; 30:S35.

17. Chapman RF, Stray-Gundersen J, Levine BD. Individual variation in response to altitude training. J Appl Physiol. 1998; 85(4):1448-56.

18. Beidleman BA, Muza SR, Rock PB, Fulco CS, Lyons TP, Hoyt RW, et al. Exercise responses after altitude acclimatization are retained during reintroduction to altitude. Med Sci Sports Exerc. 1997; 29(12): 1588-95.

19. Fulco CS, Rock PB, Cymerman A. Improving athletic performance: is altitude residence or altitude training helpful? Aviat Space Environ Med. 2000; 71(2):162-71.

20. Kayser B. Nutrition and energetics of exercise at altitude. Theory and possible practical implications. Sports Med. 1994; 17(5):309-23.

21. Wyatt FB, McCarthy JP, Neason MV, Welch BS. Comparing post exercise blood lactate between sea level and altitude natives. Med Sci Sports Exerc. 2002; 34(5 Supplement 1):S238.

22. Levine BD, Stray-Gundersen J, Duhaime G, Snell $P G$, Friedman DB. Living high-training low: the effect of altitude acclimatization/normoxic training in trained runners. Med Sci Sports Exerc. 1991; 23:25.

23. Levine BD, Stray-Gundersen J. "Living high-training low": effect of moderate altitude acclimatization with low altitude training on performance. J Appl Physiol. 1997; 83(1):102-12.

24. Meeuwsen T, Hendriksen IJM, Holewijn M. Training-induced increases in sea-level performance are enhanced by acute intermittent hypobaric hypoxia. Eur J Appl Physiol. 2001; 84(4):283-90.

25. Truijens MJ, Toussaint HM, Dow J, Levine BD. Effect of high-intensity hypoxic training on sea-level swimming performances. J Appl Physiol. 2003; 94(2):733-43. 
26. Emonson DL, Aminuddin AHK, Wight RL, Scroop GC, Gore CJ. Training-induced increases in sea-level $\mathrm{VO}_{2}$ máx are not enhanced by acute hypobaric exposure. Eur J Appl Physiol. 1997; 76(1):8-12.

27. Friedmann B, Frese F, Menold E, Kauper F, Jost J, Bartsch P. Individual variation in the erythropoietic response to altitude training in elite junior swimmers. Br J Sports Med. 2005; 39(3):148-53.

28. Stray-Gundersen J, Chapman RF, Levine BD. "Living high-training low" altitude training improves sea level performance in male and female elite runners. J Appl Physiol. 2001; 91(3):1113-20.

29. Mawson JT, Braun B, Rock PB, Moore LG, Mazzeo $\mathrm{R}$, Butterfield GE. Women at altitude: energy requirement at 4,300m. J Appl Physiol. 2000; 88(1):272-81.

30. Butterfield GE, Mazzeo R, Reeves IT, Wolfel EE, Reeves JT, Brooks GA. Exercise responses at high altitude: The Pikes Peak 1991 Experiment. Med Sci Sports Exerc. 1996; 28(5 Suppl):1.

31. Butterfield GE, Gates J, Fleming S, Brooks GA, Sutton JR, Reeves JT. Increased energy intake minimizes weight loss in men at high altitude. J Appl Physiol. 1992; 72(5):1741-8.

32. Butterfield GE. Nutrient requirements at high altitude. Clin Sports Med. 1999; 18(3):607-21.

33. Roberts AC, Reeves JT, Butterfield GE, Mazzeo RS, Sutton JR, et al. Altitude and beta-blockade augment glucose utilization during submaximal exercise. J Appl Physiol. 1996; 80(2):605-15.

34. Brooks GA, Mercier J. The balance of carbohydrate and lipid utilization during exercise: the "crossover" concept. J Appl Physiol. 1994; 76(6): 2253-61.

35. Chao W-H, Askew EW, Roberts DE, Wood SM, Perkins JB. Oxidative stress in humans during work at moderate altitude. J Nutr. 1999; 129(11): 2009-12.

36. Lawless NP, Dillard TA, Torrington KG, Davis $H Q$, Kamimori G. Improvement in hypoxemia at 4600 meters simulated altitude with carbohydrate ingestion. Aviat Space Environ Med. 1999; 70(9): 874-8.

37. Reynolds RD, Lickteig AJ, Howard MP, Deuster PA. Intakes of high fat and high carbohydrate foods by humans increased with exposure to increasing altitude during an expedition to Mt. Everest. J Nutr. 1998; 128(1):50-5.

38. Sharma A, Singh SB, Panjwani U, Yadav DK, Amitabh K, Singh S. Effect of a carbohydrate supplement on feeding behaviour and exercise in rats exposed to hypobaric hypoxia. Appetite. 2002; 39(2):127-35.

39. Askew EW. Environmental and physical stress and nutrient requirements. Am J Clin Nutr. 1995; 61(3 Suppl):631s-637s.

40. Carvalho T, Rodrigues T, Meyer F, Lancha Jr. AH, De Rose EH. Modificações dietéticas, reposição hídrica, suplementos alimentares e drogas: comprovação de ação ergogênica e potenciais riscos para a saúde - Diretriz da Sociedade Brasileira de Medicina do Esporte. Rev Bras Med Esp. 2003; 9(2):43-56.

41. Zaccaria M, Rocco S, Noventa D, Varnier M, Opocher $\mathrm{G}$. Sodium regulating hormones at high altitude: basal and post-exercise levels. J Clin Endocrinol Metab. 1998; 83(2):570-4.

42. Westerterp KR. Energy and water balance at high altitude. News Physiol Sci. 2001; 15:134-7.

43. Westerterp KR, Robach P, Woulters L, Richalet J-P. Water balance and acute mountain sickness before and after arrival at high altitude of 4,350m. J Appl Physiol. 1996; 80(6):1968-72.

44. Westerterp KR, Meijer EP, Rubbens M, Robach P, Richalet JP. Operation everest III: energy and water balance. Eur J Physiol. 2000; 439(4):483:8.

45. Singh SB, Sharma A, Yakav DK, Verma SS, Srivastava DN, Sharma KN, et al. High altitude effects on human taste intensity and hedonics. Aviat Space Environ Med. 1997; 68(12):1123-8.

46. Pfeiffer JM, Askew EW, Roberts DE, Wood SM, Freedman MS, Benson JE, et al. Effect of antioxidant supplementation on urine and blood markers of oxidative stress during extended moderate altitude training. Wilderness Environ Med. 1999; 10(2): 66-74.

47. Simon-Schnass IM. Nutrition at high altitude. J Nutr. 1992; 122(3 Suppl):778.

48. Stray-Gundersen J, Alexander C, Hochstein A, deLomos D, Levine BD. Failure of red cell volume to increase to altitude exposure in iron deficient runners. Med Sci Sports Exer. 1992; 24:S90.

49. Berglund B. High-altitude training. Aspects of haematological adaptation. Sports Med. 1992; 14(5):289-303.

Recebido em: 30/4/2004

Versão final reapresentada em: 22/4/2005

Aprovado em: 20/5/2005 